\title{
Clinical comparison of tenosynovial giant cell tumors, synovial chondromatosis, and synovial sarcoma: analysis and report of 53
}

\section{cases}

\author{
Wei Wang $^{1 \#}$, Man-Mei Long ${ }^{2 \#}$, Cheng-Jiang Wei ${ }^{1 \#}$, Xi-Wei Cui ${ }^{1}$, Jie-Yi Ren ${ }^{1}$, Yi-Hui Gu ${ }^{1}$, Qing-Feng Li $^{1}$, \\ Shun-Dong Dai ${ }^{2}$, Bin Gu ${ }^{1}$, Zhi-Chao Wang ${ }^{1}$ \\ ${ }^{1}$ Department of Plastic and Reconstructive Surgery, Shanghai Ninth People's Hospital, Shanghai Jiao Tong University School of Medicine, Shanghai, \\ China; ${ }^{2}$ Department of Pathology, Shanghai Ninth People's Hospital, School of Medicine, Shanghai Jiao Tong University, Shanghai, China \\ Contributions: (I) Conception and design: QF Li, B Gu, ZC Wang; (II) Administrative support: QF Li, B Gu, ZC Wang; (III) Provision of study \\ materials or patients: MM Long, SD Dai; (IV) Collection and assembly of data: W Wang, MM Long, CJ Wei; (V) Data analysis and interpretation: \\ W Wang, XW Cui, JY Ren, YH Gu; (VI) Manuscript writing: All authors; (VII) Final approval of manuscript: All authors. \\ "These authors contributed equally to this work. \\ Correspondence to: Shun-Dong Dai, MD. Department of Pathology, Shanghai Ninth People's Hospital, Shanghai Jiao Tong University School of Medicine, \\ 639 Zhizaoju Road, Shanghai 200011, China. Email: daishundong@qq.com; Bin Gu, MD. Department of Plastic and Reconstructive Surgery, Shanghai \\ Ninth People's Hospital, Shanghai Jiao Tong University School of Medicine, 639 Zhizaoju Road, Shanghai 200011, China. Email: gubinabc@sina.com; \\ Zhi-Chao Wang, MD, MPH. Department of Plastic and Reconstructive Surgery, Shanghai Ninth People's Hospital, Shanghai Jiao Tong University School \\ of Medicine, 639 Zhizaoju Road, Shanghai 200011, China. Email: shmuwzc@163.com; dr_wangzhichao@163.com.
}

Background: Tenosynovial giant cell tumors (TGCTs), synovial chondromatosis (SC), and synovial sarcoma (SS) exhibit similarities in clinical features and histochemical characteristics, and differential diagnosis remains challenging in clinical practice.

Methods: Data were collected from the pathology database of Shanghai Ninth People's Hospital regarding patients who underwent surgery from 2010 to 2019 with histologically confirmed TGCTs, SC, and SS. Demographic and clinicopathological data of these patients were reviewed. Immunohistochemistry staining of 14 different markers was performed. Correlation analyses of the prognoses were evaluated.

Results: A total of 26 patients with TGCTs (8 diffuse TGCTs and 18 localized TGCTs), 16 with SC, and 11 with SS were identified. Pain was the main symptom of patients with both TGCTs and SC, while a palpable mass was the most common symptom for patients with SS. In addition to clinical features, we identified vital risk factors for disease recurrence. The mean follow-up periods were 51, 39, and 14 months for TGCTs, SC, and SS, respectively. Younger patients with diffuse TGCTs or patients with a higher neutrophil/lymphocyte ratio (NLR) displayed a significantly higher frequency of recurrence. We also plotted receiver operating characteristic (ROC) curve analysis for age and NLR. The area under the ROC curve (AUC) was calculated and demonstrated the ability to distinguish recurrent from nonrecurrent cases. In addition, higher CD163 expression was linked to recurrent diffuse TGCT cases.

Conclusions: These data indicated possible characteristics of different aspects of TGCTs, SC, and SS. Further clarification and understanding of these factors will help with differential clinical diagnosis and recurrent risk assessment.

Keywords: Tenosynovial giant cell tumors (TGCTs); synovial chondromatosis (SC); synovial sarcoma (SS); differential diagnosis; prognosis

Submitted Feb 02, 2021. Accepted for publication Apr 18, 2021.

doi: $10.21037 / \mathrm{atm}-21-542$

View this article at: http://dx.doi.org/10.21037/atm-21-542

(c) Annals of Translational Medicine. All rights reserved. 


\section{Introduction}

Tenosynovial giant cell tumors (TGCTs), synovial chondromatosis (SC), and synovial sarcoma (SS) are a group of tumoral lesions arising from soft tissue and usually present with swelling or masses, pain, loss of function, or movement limitations (1). Differential diagnosis of TGCTs, SC, and SS is challenging in clinical practice due to their similar clinical manifestations, complex natural tumor histories and the limited number of patients with these lesions (2-5). However, the treatments and corresponding prognoses for the three diseases vary significantly. Longterm surveillance may be appropriate for benign TGCTs and SC. However, for SS, due to its malignant nature, early intervention is highly recommended for its high metastatic ratio. The differences in clinical treatments require a quick and accurate clinical diagnosis. In addition, high recurrence rates may exist in TGCTs, SC, and SS, especially when surgical resection cannot thoroughly proceed because their aggressive or diffuse growth should be guided by symptoms and/or disease progression (6). Early prediction of prognosis would also be helpful for more precise tumor therapies among different patients.

In the clinic, routine pathological examinations after biopsy and operation are the mainstays to provide accurate decisions for diagnosis, and they also play an irreplaceable role in determining tumor recurrence. However, many patients are hesitant to undergo invasive procedures, such as biopsy and arthroscopy. These procedures are expensive and may increase the risk of discomfort, resulting in bleeding, longer healing periods or even recurrence $(7,8)$. These procedures, though accurate, are not appropriate as a primary screen at the beginning of clinical inquiry, especially if malignancy is a possibility. In contrast, clinical information collected from medical histories, laboratory investigations and radiologic examinations can help classify, exclude and ascertain the diagnosis of one specific disease or a disease with similarities in certain aspects (9). In addition, information can guide the evaluation of prognosis and provide suggestions about the frequency of follow-up. However, given that TGCTs, SC, and SS are a relatively rare group of tumors, there are limited previous clinical analyses for distinguishing these three tumors.

In this study, we aimed to illustrate the clinical features of TGCTs, SC, and SS in detail, discuss the differential diagnosis, and evaluate the outcomes after surgical treatment. Because of the differences in growth behavior, TGCTs are further classified into localized TGCTs
(1-TGCTs) and diffuse TGCTs (d-TGCTs) for more precise comparison. Before our research, these three entities were mainly covered in the English literature as case reports or a small case series. This is the first analysis comparing the clinical features of various aspects of TGCTs, SC, and SS in a relatively large patient cohort considering the rarity of these tumors. We also analyzed whether there are any possible clinical or pathological-based signatures to predict recurrence risk after surgery to provide insights for postoperative therapy. By combining clinical information for primary differential diagnosis and pathology analysis for prognosis prediction, we hope this study will assist in clinical diagnosis, applying precise treatments and providing reliable suggestions for follow-up care.

We present the following article in accordance with the STROBE reporting checklist (available at http://dx.doi. org/10.21037/atm-21-542).

\section{Methods}

\section{Sample selection}

Tumor samples of TGCTs, SC, and SS were selected from the tissue bank of the Institute of Pathology, Shanghai Ninth People's Hospital, Shanghai Jiao Tong University School of Medicine, from 2010 to 2019. All samples that were histologically proven as TGCTs, SC, and SS by the World Health Organization (WHO) criteria (10) were included. All specimens included in the study were from initial tumor resection. No recurrent tumor specimens were included in the further staining analysis. Moreover, patients with multiple hospitalizations (first biopsy and then surgery) were counted only once. The study was conducted in accordance with the Declaration of Helsinki (as revised in 2013). This study was approved by the local ethics committee of the Shanghai Ninth People's Hospital, Shanghai Jiao Tong University School of Medicine (No. SH9H-2019-T163-2), and individual consent for this retrospective analysis was waived.

\section{Clinical data}

All the data were obtained from the electronic clinical records, including age, sex, chief complaint, lesion site, neoplastic size, related surgery or injury history, laboratory results, surgery type, and follow-up. Tumor volumes (TVS) were calculated using Gobel's method as described previously: TVS $=\pi / 6 \times$ (tumor length $) \times($ tumor width $)$ 
$\times$ (tumor depth) (11). The neutrophil/lymphocyte ratio (NLR) was defined as the ratio of absolute neutrophil counts divided by absolute lymphocyte counts as prognostic markers (12).

\section{Histologic features}

In addition to $\mathrm{H} \& \mathrm{E}$ staining, immunohistochemistry was performed on 4- $\mu \mathrm{m}$-thick formalin-fixed paraffin-embedded tissue sections. Briefly, after dewaxing and rehydration at $60^{\circ} \mathrm{C}$, the sections were treated with citrate buffer in a microwave oven for $30 \mathrm{~min}$ to retrieve nuclear antigens. The tissue sections were then blocked for endogenous peroxidase activity and incubated overnight at $4{ }^{\circ} \mathrm{C}$ with antibodies. The recommended dilution ratio was used for all of the antibodies. Secondary antibodies were added for an hour at room temperature (Maxim, China), and antibody detection was revealed by the DAB substrate (Vector Laboratories, USA), followed by costaining with hematoxylin solution (Sangon, China). The results and previous $\mathrm{H} \& \mathrm{E}$ staining slides were reviewed and evaluated at low magnification by two expert pathologists. The staining was scored as 4+ (positive in greater than $50 \%$ of cells), $3+$ (positive in $25-50 \%$ of cells), $2+$ (positive in $5-25 \%$ of cells), $1+$ (only weak reactivity or less than $5 \%$ of positive cells), or 0 (no reactivity).

\section{Statistical analysis}

Data variables were summarized as percentages or means \pm standard deviations (SDs). Given the small sample size, the statistical analysis was performed with the nonparametric, 2-tailed Mann-Whitney test by Prism 6, GraphPad (La Jolla, CA, USA). A P value less than 0.05 was considered statistically significant. The prognostic accuracy was further evaluated by the area under the curve (AUC) of the receiver operating characteristic (ROC) curve.

\section{Results}

\section{Patient characteristics}

The baseline clinical characteristics of the included participants who were histologically documented as having TGCTs, SC, and SS between 2010 and 2019 are listed in Table 1. There were 26 TGCT samples ( 8 diffuse TGCTs and 18 localized TGCTs), 16 SC samples, and $11 \mathrm{SS}$ samples from patients with median ages of 54.5, 62.0, and
24.0, respectively. The common localizations of the TGCT, SC and SS samples were the knee and lower extremity. The sex distribution was nearly equal in the SC and SS samples, and a female preponderance was found among TGCT samples. Patients with SS had the timeliest diagnosis in the hospital among the three diseases, with an average disease duration before diagnosis of $1.2 \pm 2.9$ years. A total of 12 of the 26 TGCT patients had tumors larger than $5 \mathrm{~cm}$, with an average volume of $110.1 \mathrm{~cm}^{3}$, the largest size among the three. The mean tumor volumes of SC and SS were 18.6 and $46.5 \mathrm{~cm}^{3}$, respectively. Typical images acquired from radiology and H\&E staining are presented in Figure 1.

\section{Chief complaints for clinical inquiry}

We also reviewed the chief complaints from patients with these tissue tumors. The detailed data are presented in Table 2. In addition to a palpable mass (17/53), pain (30/53), limited movement (15/53), swelling (13/53), and numbness (2/53) were among the most common complaints of patients in the clinic. Pain was the main symptom of both TGCTs and SC, while a mass was the main symptom of SS. In other words, a patient with a chief complaint of pain was more likely to be diagnosed with TGCTs or SC, which were more likely to be benign tumors. However, patients with less pain and seemingly mild clinical symptoms were more likely to have malignant SS.

\section{Surgical methods, complications, outcomes, and prognosis}

For TGCTs and SC, both open and arthroscopic surgeries are commonly used. Open surgery was more frequently used for TGCTs due to their large volume and aggressive nature, especially when dealing with the diffuse type. All the cases of SS were completely resected with wide margins. Two young d-TGCT patients, aged 26 and 28, experienced postoperative ankylosis after open synovectomy. All discharged patients were recommended for joint function rehabilitation 1 month after surgery. Four patients with d-TGCTs in the knees suffered recurrence. After standard resection ( 3 open resections and 1 arthroscopy), the tumor relapsed, and patients acknowledged symptom-induced (2 for swollen joints and 1 for pain) hospital checks or annual physical examinations. Among these four patients with recurrence, only one underwent a further knee arthroplasty in April 2020. Recurrent SC was found in 3 patients with original lesions in the knee, hip and ankle. All of them were initially treated with arthroscopic synovectomy and felt 
Table 1 Comparison of clinical characteristics related with TGCT, SC and SS

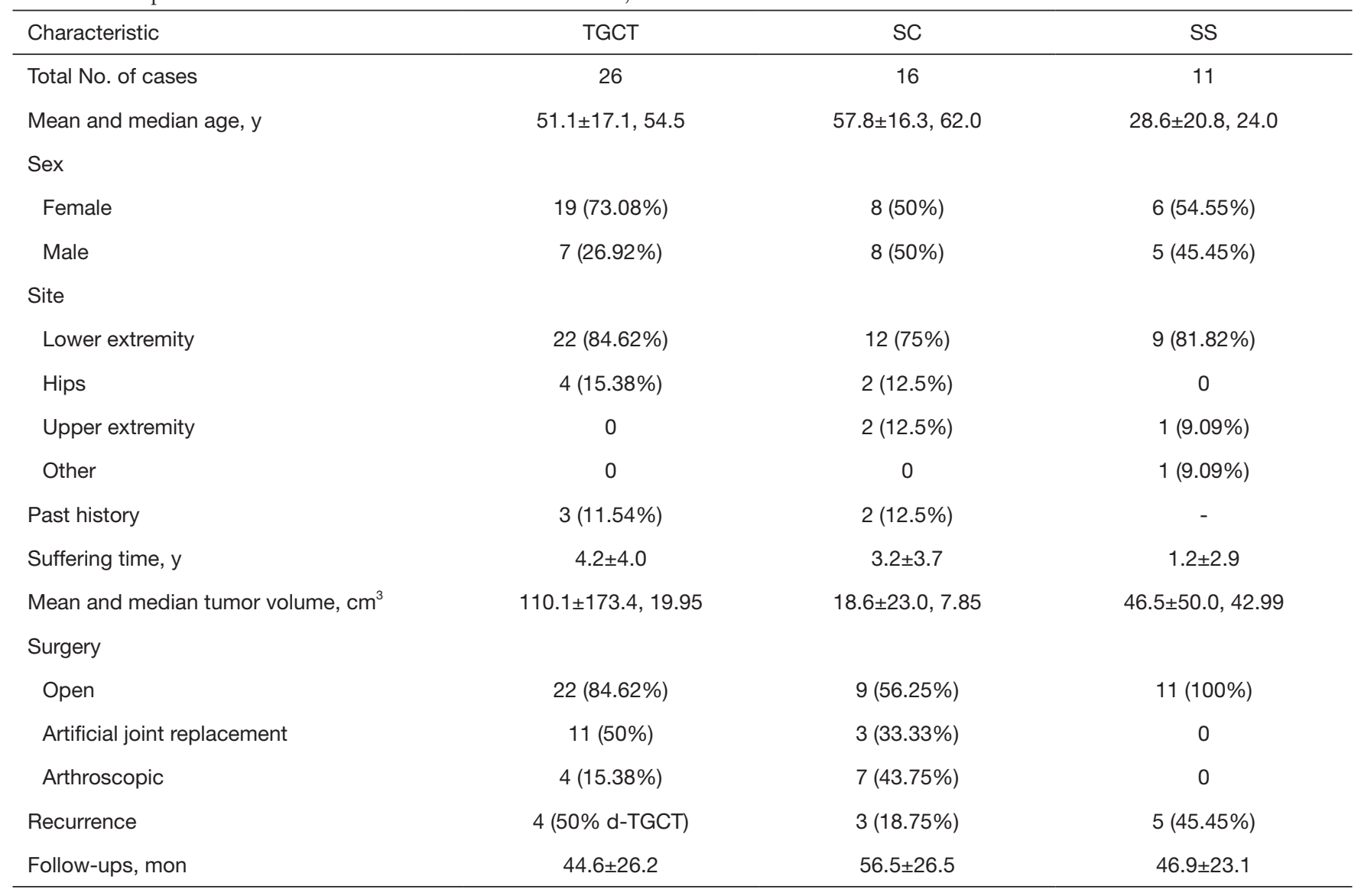

TGCTs, tenosynovial giant cell tumors; d-TGCTs, diffuse tenosynovial giant cell tumors; SC, synovial chondromatosis; SS, synovial sarcoma.

pain in the same operated joint. One patient underwent total artificial knee arthroplasty three years later, and the pain was relieved. SS patients have a greater susceptibility $(45.45 \%, 5 / 11)$ to recurrence than the other two tumor types due to its malignant nature. All these recurrent lesions were from the lower extremities. Further treatments, including chemical/radiological agents ( 2 cases) and tumor enlargement resection (2 cases), were performed. No signs of recurrence or lung metastases were found at the SS follow-up.

All possible prognostic factors of recurrence in these three diseases were considered (Table 3). The results indicated that each identified clinical signature had a better predictive value as a risk factor for d-TGCTs than for the other tumors. More precisely, younger d-TGCT patients had a higher risk for recurrence $(\mathrm{P}=0.029)$, and a higher NLR was another risk factor in patients with d-TGCTs
$(\mathrm{P}=0.048)$ but not in those with $\mathrm{SC}$ or SS. Tumor size, in our research, showed no predictive value among TGCTs, SC, and SS. We then evaluated the combination of patient age and NLR index to judge the prognostic performance by using ROC curve analysis. The AUC was calculated to be 0.7475 , as shown in Figure 2, demonstrating that this combination had essential clinical significance to distinguish recurrent from nonrecurrent cases among these three diseases. In addition to clinical variables, we also compared the IHC staining intensity of several markers and found the CD163 expression level to be a prognostic marker of recurrence in d-TGCTs.

Although all these patients (53/53, 100\%) showed different levels of positive CD163 immunoreactivity (Table 4 and Figure 3), a higher CD163 expression level was significantly related to a higher recurrence rate in patients with d-TGCTs $(\mathrm{P}=0.027)$. The association between CD163 

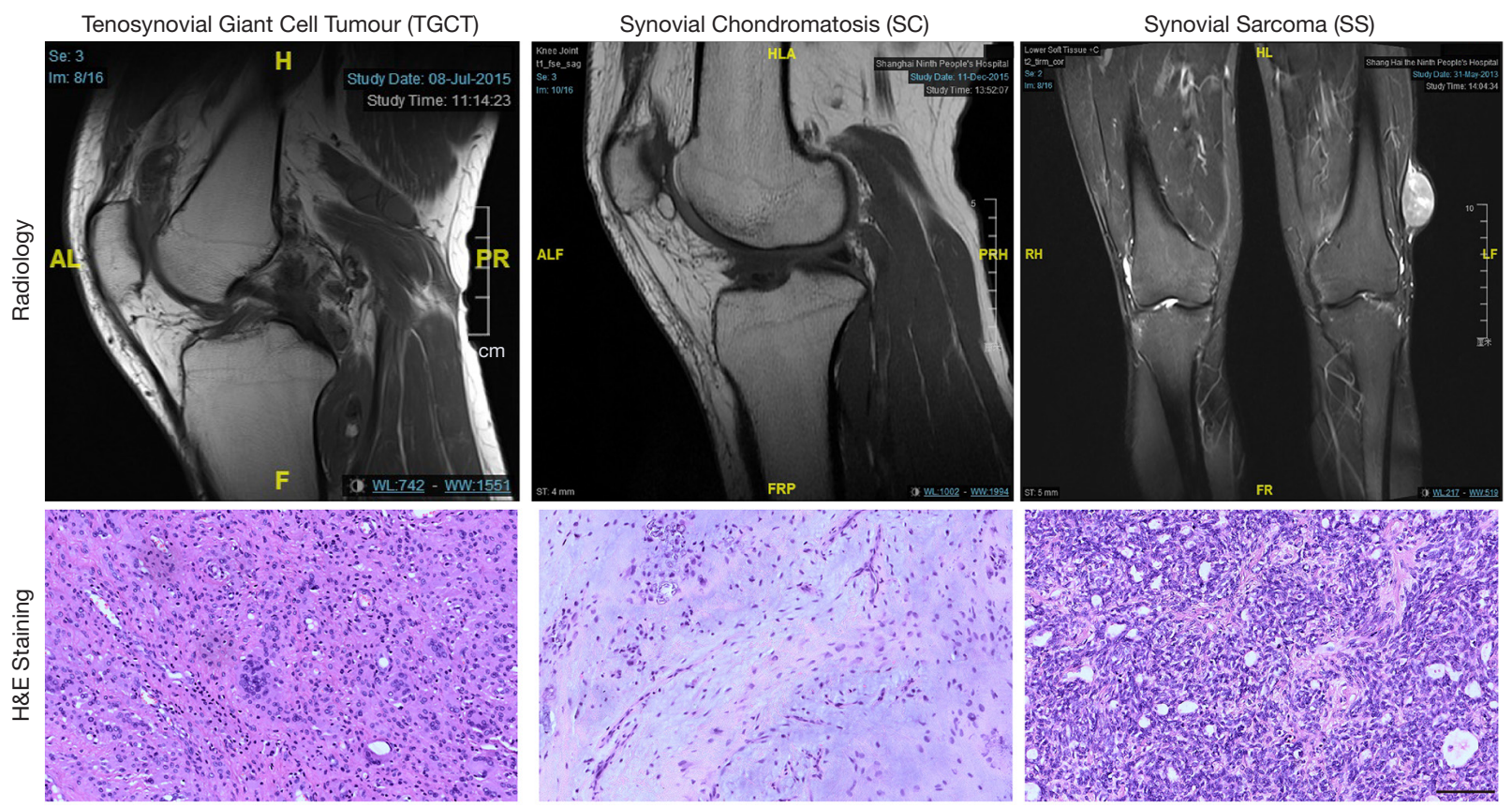

Figure 1 Typical presentations from radiological images and H\&E staining of tenosynovial giant cell tumors (TGCTs), synovial chondromatosis (SC) and synovial sarcoma (SS). Scale bars, $100 \mu \mathrm{m}$.

Table 2 The main symptoms of patients with TGCT, SC and SS for hospitalization

\begin{tabular}{lccc}
\hline Main symptoms & TGCT (\%) & SC (\%) & SS (\%) \\
\hline Tumor & $5(19.23)$ & $3(18.75)$ & $9(81.82)$ \\
Pain & $16(61.54)$ & $10(62.5)$ & $4(36.36)$ \\
Limited movement & $11(42.31)$ & $3(18.75)$ & $1(6.25)$ \\
Swelling & $10(38.46)$ & $3(18.75)$ & 0 \\
Numbness & 0 & 0 & $2(12.5)$ \\
\hline
\end{tabular}

TGCT, tenosynovial giant cell tumor; SC, synovial chondromatosis; SS, synovial sarcoma.

staining and recurrence was based on initial diagnosis. However, there was no other specific expression linkage between positive CD163 staining and the recurrence of SC or SS. Other common immunohistochemical biomarkers of pathological evaluation in 6 nonrecurrent SS patients and 4 recurrent SS patients are listed in Table 5, including c-Myc, BMP-6, Gal-8, vimentin, CK, CK7, CK19, EMA, SMA, Bcl-2, calponin and Ki67.

\section{Discussion}

Although TGCTs and SC are benign tumors, they both have high local recurrence rates post-operation. A total of $9.8 \%$ of patients with 1-TGCTs and $19.1 \%$ of patients with d-TGCTs suffered disease recurrence in a large Denmark cohort study (13). High local recurrence rates have also been found in SC after arthroplasty. Recurrence occurred in 3/26 (11\%) SC patients in hips and 5/19 (27\%) in knees $(14,15)$. These tumors can grow aggressively and extra-articularly $(16,17)$. Rarely, they may coexist with or transform into malignant sarcoma (Figure 4) (18). We summarized the English literature from PubMed about the clinical presentation of their malignant changes in Table 6 to increase the understanding of this process $(16,18-22)$.

Understanding the differences among TGCTs, SC, and SS is essential for diagnostic, prognostic, and therapeutic 
Table 3 A summary of relapse according to clinical and biologic characteristics of patients with TGCT, SC and SS

\begin{tabular}{|c|c|c|c|c|c|c|c|}
\hline Variable & $\frac{\text { I-TGCT }}{\text { Non- }}$ & \multicolumn{2}{|c|}{ d-TGCT } & \multicolumn{2}{|c|}{ SC } & \multicolumn{2}{|c|}{ SS } \\
\hline No. & 18 & 4 & 4 & 13 & 3 & 6 & 5 \\
\hline Age & $55.76 \pm 16.99$ & $54.25 \pm 4.65$ & $33.75 \pm 13.52$ & $56 \pm 18.12$ & $57 \pm 13.75$ & $36.57 \pm 23.16$ & $26.6 \pm 25.93$ \\
\hline$<50$ & 7 (38.89\%) & 0 & $3(75 \%)$ & $5(38.46 \%)$ & $1(33.33 \%)$ & $3(50 \%)$ & $4(80 \%)$ \\
\hline $\mathrm{P}$ & & \multicolumn{2}{|c|}{0.029} & \multicolumn{2}{|c|}{ NS } & \multicolumn{2}{|c|}{ NS } \\
\hline Volume & $124.2 \pm 201.9$ & $77.14 \pm 55.92$ & $123.4 \pm 134.7$ & $11.99 \pm 16.88$ & $24.33 \pm 36.08$ & $42.44 \pm 29.64$ & $63.81 \pm 75.95$ \\
\hline$<5$ & 12 (66.67\%) & $2(50 \%)$ & $1(25 \%)$ & 12 (92.31\%) & 2 (66.67\%) & $4(66.67 \%)$ & $1(20 \%)$ \\
\hline$>5$ & $6(33.33 \%)$ & 2 (50\%) & 3 (75\%) & $1(7.69 \%)$ & $1(33.33 \%)$ & 2 (33.33\%) & $4(80 \%)$ \\
\hline$<2$ & 7 (43.75\%) & $1(25 \%)$ & 1 (33.33\%) & $1(12.5 \%)$ & $2(66.67 \%)$ & $4(66.67 \%)$ & $4(80 \%)$ \\
\hline$>2$ & $9(56.25 \%)$ & 3 (75\%) & 2 (66.67\%) & 7 (87.5\%) & $1(33.33 \%)$ & 2 (33.33\%) & $1(20 \%)$ \\
\hline $\mathrm{P}$ & & \multicolumn{2}{|c|}{0.048} & \multicolumn{2}{|c|}{ NS } & \multicolumn{2}{|c|}{ NS } \\
\hline
\end{tabular}

TGCTs, tenosynovial giant cell tumors; I-TGCT, localized tenosynovial giant cell tumor; d-TGCTs, diffuse tenosynovial giant cell tumors; SC, synovial chondromatosis; SS, synovial sarcoma.

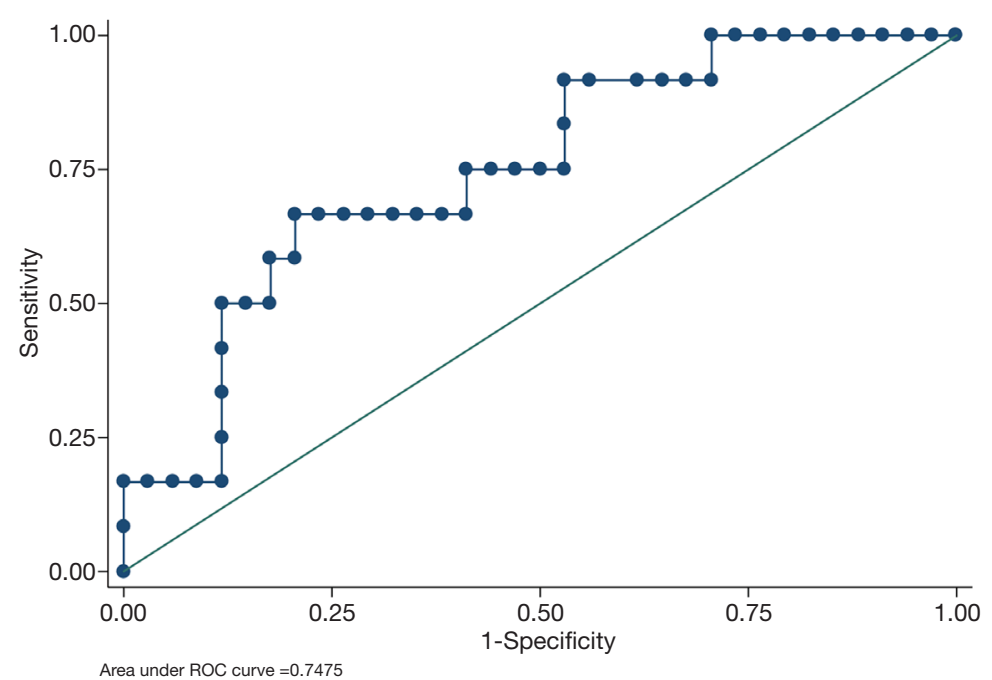

Figure 2 Receiver operating characteristic (ROC) curve analysis for the prediction of recurrence in tenosynovial giant cell tumors (TGCTs), synovial chondromatosis (SC) and synovial sarcoma (SS).

implications. In this article, we analyzed their differences from several aspects, which may provide diagnostic information for primary care physicians when determining the possible disease in patients; therapeutic information when determining the necessity for further examination with a more active treatment such as biopsy or surveillance or whether conservative treatment itself is sufficient; and prognostic information for evaluating the probability 
Table 4 Summary of CD163 immunohistochemical staining

\begin{tabular}{|c|c|c|c|c|c|c|c|}
\hline Variable & $\frac{\text { I-TGCT }}{\text { Non- }}$ & \multicolumn{2}{|c|}{ d-TGCT } & \multicolumn{2}{|c|}{ SC } & \multicolumn{2}{|c|}{ SS } \\
\hline No. & 18 & 4 & 4 & 13 & 3 & 6 & 5 \\
\hline $4+(\%)$ & $4(22.22 \%)$ & 0 & $3(75 \%)$ & 0 & 0 & $1(16.67 \%)$ & 0 \\
\hline $3+(\%)$ & $9(50 \%)$ & $2(50 \%)$ & $1(25 \%)$ & 4 (30.77\%) & 0 & $3(50 \%)$ & $1(20 \%)$ \\
\hline $1+(\%)$ & 1 (5.56\%) & $2(50 \%)$ & 0 & 2 (15.38\%) & 1 (33.33\%) & 2 (33.33\%) & 2 (40\%) \\
\hline 0 & 0 & 0 & 0 & 0 & 0 & 0 & 0 \\
\hline$P$ & & 0.027 & & NS & & NS & \\
\hline
\end{tabular}

I-TGCT, localized tenosynovial giant cell tumor; d-TGCTs, diffuse tenosynovial giant cell tumors; SC, synovial chondromatosis; SS, synovial sarcoma.

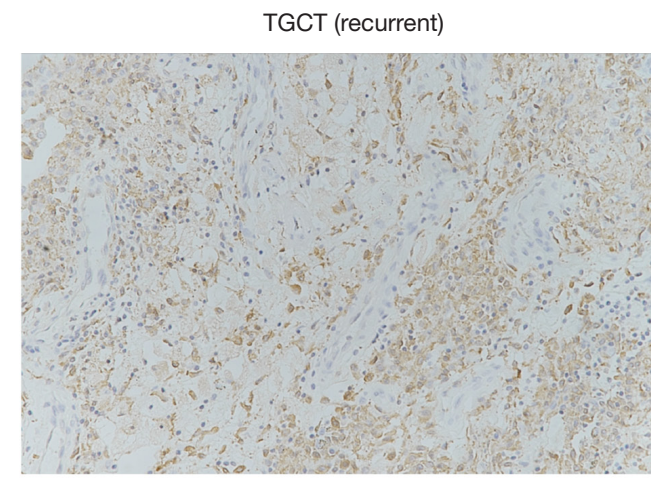

SC

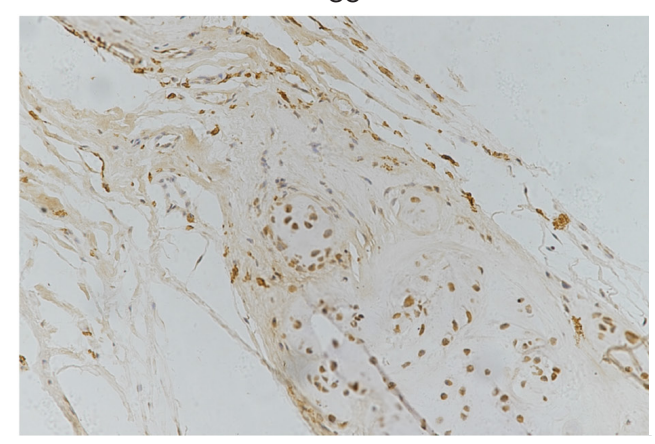

TGCT (non-recurrent)

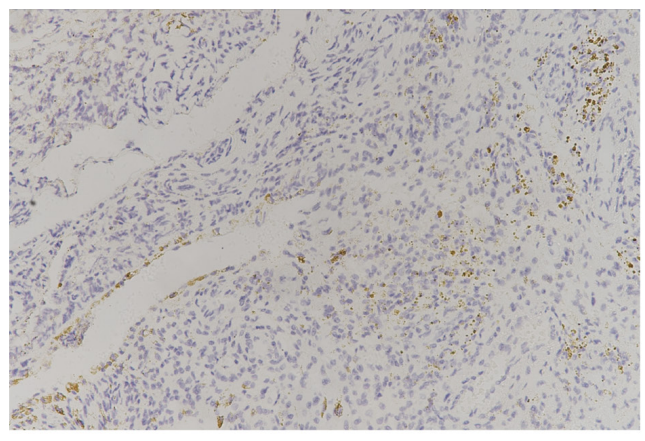

SS

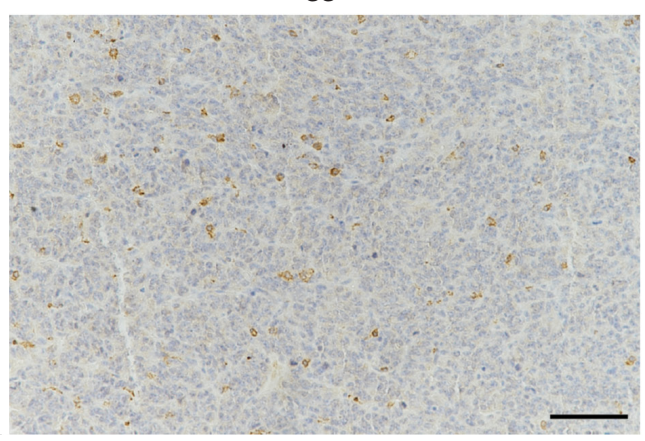

Figure 3 Representative CD163 immunohistochemistry sections of tenosynovial giant cell tumors (TGCTs) (recurrent and nonrecurrent cases), synovial chondromatosis (SC) and synovial sarcoma (SS). Scale bars, $100 \mu \mathrm{m}$.

of recurrence and suggesting follow-up frequency after hospital discharge.

Among the 53 patients in our study, 26 TGCTs, 16 SC, and 11 SS were unanimously labeled by 2 soft-tissue pathologists based on microscopic histological grounds. Some different presentations in our findings compared with those of other research are listed as follows. First, in terms of diagnostics, the results of our research showed that the average age of TGCT patients was 51.12 years old, which was higher than the age of those with SC and SS. Ottaviani $\mathrm{S}$ and colleagues reported contradictory results: that TGCTs occur predominantly occur young adults (23). 
Page 8 of 13

Wang et al. Clinical comparison of TGCT, SC and SS

Table 5 Immunohistochemical results of non-recurrent and recurrent cases of SS

\begin{tabular}{|c|c|c|c|c|c|c|c|c|c|c|}
\hline Variable & \multicolumn{6}{|c|}{ Non-recurrent } & \multicolumn{4}{|c|}{ Recurrent } \\
\hline C-Myc & + & + & + & - & + & - & + & - & + , weak & + , weak \\
\hline BMP-6 & - & - & - & - & + & - & - & - & - & - \\
\hline Gal-8 & - & - & - & - & - & - & - & + & - & - \\
\hline CK & + & - & + , epithelial & & + & - & - & - & - & - \\
\hline CK7 & + & & + , epithelial & - & + & & - & - & - & - \\
\hline CK19 & + & & + , epithelial & - & + & - & - & - & - & - \\
\hline EMA & + & - & + , epithelial & + & + & - &,+ partial &,+ focal & + & + \\
\hline Calponin & + , partial & - &,+ partial & - & - & - & & + & + & + \\
\hline Ki67 & $40 \%$ & $40 \%$ & $30 \%$ & $35 \%$ & & - & $40 \%$ & $5 \%$ & $2 \%$ & $2 \%$ \\
\hline
\end{tabular}

SS, synovial sarcoma.

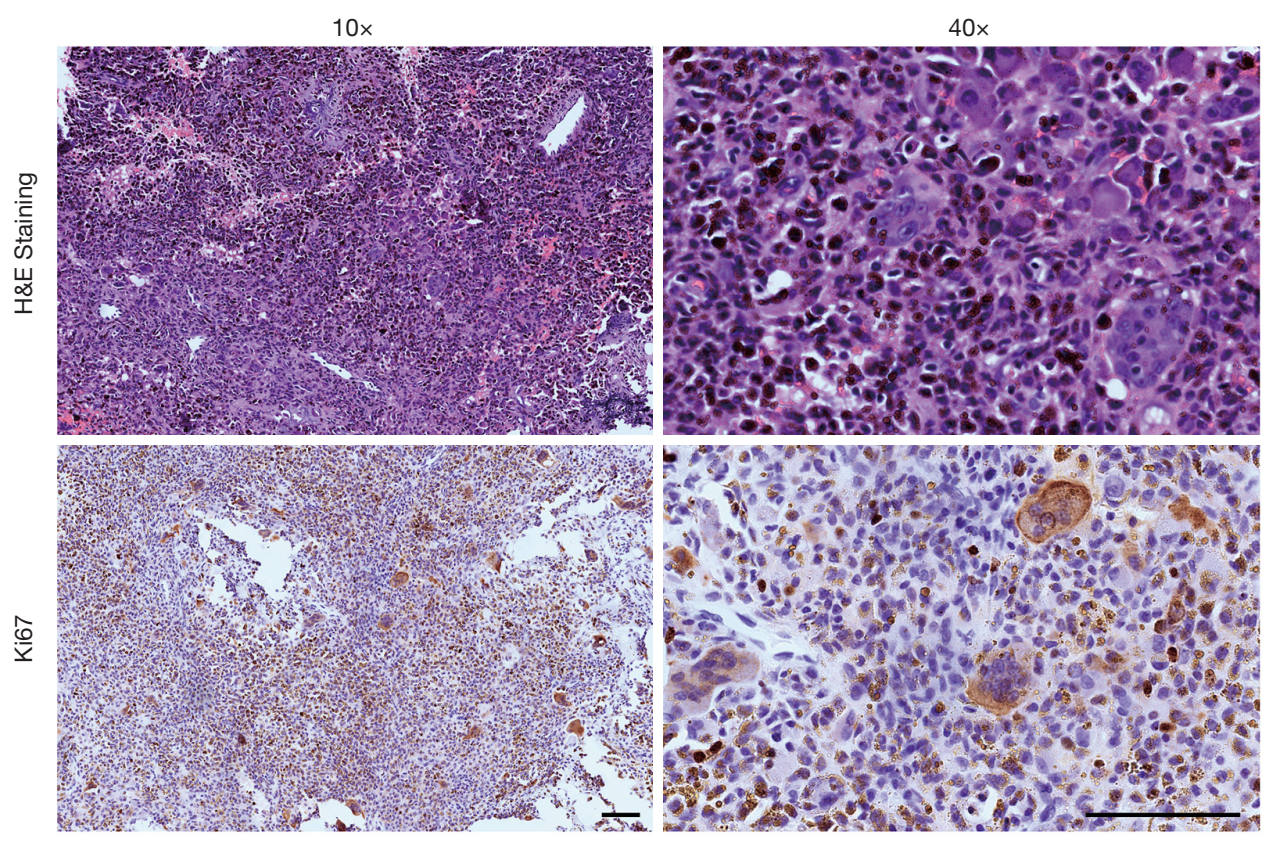

Figure 4 H\&E staining and Ki67 immunohistochemistry results of malignant tenosynovial giant cell tumor (TGCT). Scale bars, 100 um.

This difference may be because most of our patients were diagnosed incidentally after knee arthroplasty operation as $\mathrm{OA}$ on admission, which agrees with the conclusions of other studies $(18,24)$. The relatively balanced sex ratio in SC patients is also different from the reported male prevalence (25). In terms of clinical symptoms, the fundamental chief complaint was pain in those with TGCTs and SC and a palpable mass in those with SS. 
Table 6 Clinical features of malignant TGCT and SC summarized from literatures

\begin{tabular}{|c|c|c|}
\hline Variable & Malignant TGCT & Malignant SC \\
\hline Total No. of cases & 57 & 72 \\
\hline Mean age, y & $51.16 \pm 17.33$ & $46.23 \pm 14.75$ \\
\hline \multicolumn{3}{|l|}{ Sex } \\
\hline Female & $31 / 57(54.4 \%)$ & $27 / 65(41.5 \%)$ \\
\hline Male & $26 / 57(45.6 \%)$ & $38 / 65(58.5 \%)$ \\
\hline \multicolumn{3}{|l|}{ Site } \\
\hline Lower extremity & 35 & 36 \\
\hline Hips & 9 & 27 \\
\hline Upper extremity & 8 & 7 \\
\hline Other & 4 & 2 \\
\hline Metastases & $31 / 57(54.4 \%)$ & 19/61 (31.1\%) \\
\hline Lung & 21 & 18 \\
\hline Lymph node & 12 & 0 \\
\hline Groin \& pelvis & 0 & 0 \\
\hline Brain & 4 & 3 \\
\hline Surgery & 41 & 61 \\
\hline Resection & 26 & 14 \\
\hline Amputation & 12 & 35 \\
\hline Radiation/chemotherapy & 12 & 9 \\
\hline Artificial joint replacement & 1 & 3 \\
\hline Recurrence & $27 / 56(48.2 \%)$ & $21 / 57(36.8 \%)$ \\
\hline
\end{tabular}

$(16,18-22)$. TGCT, tenosynovial giant cell tumor; SC, synovial chondromatosis.

Numbness was more common in SS and described in two of our patients, which may be due to nerve compression caused by extra-articular growth and rapid tumor growth. This symptom may exist more frequently in SS due to its aggressive growth behaviors, but it is also reported in malignant TGCTs $(4,26)$ and SCs $(27,28)$. The high FDG uptake in PET/CT examination of malignant tumors such as SS can also exist in TGCTs, which increases the difficulty of diagnosis.

Second, we studied possible factors related to recurrence and prognosis. Accurately identifying patients with a high risk of recurrence is crucial for improving prognosis, as these patients might benefit from early adjuvant chemotherapy or targeted therapy with a more frequent follow-up. Previously, studies have found several indexes, including age, tumor size and preoperative NLR, as effective predictors for evaluating the relapse of TGCTs (29), SS $(30,31)$ or other highly recurrent neoplasms $(32,33)$. In our research, we found that the age of onset and preoperative NLR were both strongly associated with the long-term prognosis of d-TGCTs. This suggests a closer follow-up for young TGCT patients with the diffuse tumor type. Moreover, the combination of age and NLR may further help to predict recurrence and outcomes for these three soft tissue tumors with a high AUC value (0.7475) by using ROC curve analysis. Young SC and SS patients with larger tumor sizes are also prone to a higher recurrence risk, although there was no significant difference. We also summarized the recent 5 years of recurrence factors of these three diseases in Table 7 (34-42). In addition to clinical data that provide a thorough outline of the diseases, pathological results remain the gold standard for disease diagnosis and classification. To systematically evaluate the distribution differences of clinicopathological characteristics between these diseases, we compared the expression of several other IHC markers in addition to the conventional markers of vimentin, CK, and EMA. The expression of CD163, c-Myc, and gal- 8 was calculated in the included cases, as they are correlated with tumor aggression and poor outcomes in other cancers (43-45). As a macrophage marker, CD163 was used to detect scavenger receptors and was positive in synovial cells of TGCTs (46). In our patients, significantly higher CD163 expression was observed, especially in recurrent cases of d-TGCTs, which was consistent with previous studies that pointed out the relationship between the excessive proliferative process or even tumor progression of TGCTs and CD163 expression levels $(47,48)$. A better prognosis for SS may also be linked to the negative expression of c-Myc (49), though this was not proven in our data.

Third, systemic therapy is needed to achieve long disease-free situations. For severe or refractory TGCT cases, further adjuvant therapies, including external beam radiotherapy, intra-articular injection of medications, or targeted therapy with pexidartinib are promising $(4,50,51)$. However, by telephone review, we found that the number of patients who received combined therapy after surgery was low. According to our results, only one patient with a d-TGCT $(1 / 8,12.5 \%)$ underwent regular external beam radiotherapy and did not relapse after 33 months of followup; others who underwent regular annual CT examinations had a 4/7 (57.14\%) recurrence ratio. Accurately identifying 
Table 7 Recent 5 years studies about the recurrence risk of TGCTs, SC and SS

\begin{tabular}{|c|c|c|c|c|c|}
\hline Disease & Factors & No. of cases & Recurrence & Conclusion & Reference \\
\hline \multirow{3}{*}{ TGCTs } & Incomplete resection & $33(15 \mathrm{l}-,, 18 \mathrm{~d}-)$ & $10 / 33(30 \%)$ & Increase local recurrence in hindfoot TGCT & $(36)$ \\
\hline & $\begin{array}{l}\text { Tumor size and } \\
\text { arthroscopy }\end{array}$ & 941 & $100 / 823(12 \%)$ & $\begin{array}{l}\text { Increase recurrence when I-TGCTs } \geq 5 \mathrm{~cm} \text { and } \\
\text { initial arthroscopy treatment }\end{array}$ & $(38)$ \\
\hline & None & 1,192 & $425 / 966(44 \%)$ & $\begin{array}{l}\text { risk factors for recurrent d-TGCT were not } \\
\text { identified }\end{array}$ & (39) \\
\hline \multirow{2}{*}{ SS } & $\begin{array}{l}\text { Size and adjuvant } \\
\text { therapy }\end{array}$ & 60 & 8/60 (13.3\%) & $\begin{array}{l}\text { Increase morbidity and mortality when adjuvant } \\
\text { chemo/radiotherapy applied in } \leq 5 \mathrm{~cm} \text { patient }\end{array}$ & $(41)$ \\
\hline & $\begin{array}{c}\text { Tumor re-excision } \\
\text { and trained oncologic } \\
\text { surgeon }\end{array}$ & 63 & 7/63 (11.1\%) & $\begin{array}{l}\text { Decrease recurrence when re-excising tumor by } \\
\text { oncologic surgeon }\end{array}$ & $(42)$ \\
\hline
\end{tabular}

TGCT, tenosynovial giant cell tumor; SC, synovial chondromatosis; SS, synovial sarcoma. I-, localized-; d-, diffuse-.

patients with a high risk of recurrence is crucial to improve prognosis, as these patients might benefit from early systemic therapy, including adjuvant chemotherapy or targeted therapy.

This retrospective analysis has limitations. (I) These three diseases are not common, and the incidence of malignant changes in TGCTs and SC is extremely rare. The results of this research may not be as practical in the clinic as other diseases. (II) The sample size was relatively small because of the low incidence rate of soft tissue neoplasms. (III) This analysis lacked systematic functional outcome evaluations for assessing the recovery of joints from the operation. The further inclusion of patients in the future might contribute to a better understanding of the differences and prognosisrelated factors among these three diseases. However, this study still exhibited several important results. We believe the presented information will provide valuable information for a better understanding of the differential diagnosis of these soft tissue tumors.

\section{Conclusions}

In summary, TGCTs, SC, and SS can have similar clinical manifestations that sometimes make diagnosis difficult. Further imaging examination or biopsy for pathology is needed when features are atypical. This article helps to understand the differences among these three rare soft tissue tumors in terms of various aspects, including clinical characteristics, main symptoms, laboratory data, histological features, and prognostic outcomes. It also provides practical suggestions for the close follow-up of d-TGCT patients when they are young, have a high preoperative NLR or have strong CD163 expression in initial tumor sections.

\section{Acknowledgments}

Funding: This work was supported by grants from Youth Doctor Collaborative Innovation Team Project (QC201803) of Shanghai Ninth People's Hospital, Shanghai Jiaotong University School of Medicine; the Shanghai Youth Top-Notch Talent Program (201809004), "Chenguang Program" supported by Shanghai Education Development Foundation and Shanghai Municipal Education Commission (19CG18) and Science and Technology Commission of Shanghai Municipality (19JC1413), Shanghai Rising Star Program (20QA1405600), Innovative 
research team of high-level local universities in Shanghai (SSMU-ZDCX20180700), Shanghai Municipal Key Clinical Specialty (shslczdzk00901), National Natural Science Foundation of China (81802937).

\section{Footnote}

Reporting Checklist: The authors have completed the STROBE reporting checklist. Available at http://dx.doi. org/10.21037/atm-21-542

Data Sharing Statement: Available at http://dx.doi. org/10.21037/atm-21-542

Peer Review File: Available at http://dx.doi.org/10.21037/ atm-21-542

Conflicts of Interest: All authors have completed the ICMJE uniform disclosure form (available at http://dx.doi. org/10.21037/atm-21-542). ZCW and WW serve as an unpaid section editor of Annals of Translational Medicine from Oct 2019 to Dec 2021. The other authors have no conflicts of interest to declare.

Ethical Statement: The authors are accountable for all aspects of the work in ensuring that questions related to the accuracy or integrity of any part of the work are appropriately investigated and resolved. The study was conducted in accordance with the Declaration of Helsinki (as revised in 2013). The study was approved by ethics committee of the Shanghai Ninth People's Hospital, Shanghai Jiao Tong University School of Medicine (No. SH9H-2019-T163-2) and individual consent for this retrospective analysis was waived.

Open Access Statement: This is an Open Access article distributed in accordance with the Creative Commons Attribution-NonCommercial-NoDerivs 4.0 International License (CC BY-NC-ND 4.0), which permits the noncommercial replication and distribution of the article with the strict proviso that no changes or edits are made and the original work is properly cited (including links to both the formal publication through the relevant DOI and the license). See: https://creativecommons.org/licenses/by-nc-nd/4.0/.

\section{References}

1. Levine BD, Motamedi K, Seeger LL. Synovial Tumors and Proliferative Diseases. Rheum Dis Clin North Am 2016;42:753-68.

2. Rebuzzi SE, Grassi M, Catalano F, et al. Multiple systemic treatment options in a patient with malignant tenosynovial giant cell tumour. Anticancer Drugs 2020;31:80-4.

3. Lindberg F, Lund B, Normark S. Gene products specifying adhesion of uropathogenic Escherichia coli are minor components of pili. Proc Natl Acad Sci U S A 1986;83:1891-5.

4. Theunissen CI, Bras J, Lienden KP, et al. Malignant giant cell tumor in the carpal tunnel: a case report and review of literature. J Wrist Surg 2013;2:271-5.

5. Vogel D, Walsh ME, Chen JT, et al. Comparison of vein valve function following pharmacomechanical thrombolysis versus simple catheter-directed thrombolysis for iliofemoral deep vein thrombosis. J Vasc Surg 2012;56:1351-4.

6. Staals EL, Ferrari S, Donati DM, et al. Diffuse-type tenosynovial giant cell tumour: Current treatment concepts and future perspectives. Eur J Cancer 2016;63:34-40.

7. Schepis F, Turco L, Bianchini M, et al. Prevention and Management of Bleeding Risk Related to Invasive Procedures in Cirrhosis. Semin Liver Dis 2018;38:215-29.

8. Irtan S, Van Tinteren H, Graf N, et al. Evaluation of needle biopsy as a potential risk factor for local recurrence of Wilms tumour in the SIOP W'T 2001 trial. Eur J Cancer 2019;116:13-20.

9. Abdelkader A, Hunt B, Hartley CP, et al. Cystic Lesions of the Pancreas: Differential Diagnosis and Cytologic-Histologic Correlation. Arch Pathol Lab Med 2020;144:47-61.

10. Fletcher CDM, World Health Organization. International Agency for Research on Cancer. WHO classification of tumours of soft tissue and bone. 4th ed. Lyon: IARC Press, 2013:468.

11. Yao H, Wang B, Wen L, et al. Comparison of clinical features, management and outcomes of osteosarcoma located in proximal fibula and proximal tibia: a propensity score matching analysis. BMC Cancer 2018;18:1195.

12. Diem S, Schmid S, Krapf M, et al. Neutrophil-toLymphocyte ratio (NLR) and Platelet-to-Lymphocyte ratio (PLR) as prognostic markers in patients with nonsmall cell lung cancer (NSCLC) treated with nivolumab. Lung Cancer 2017;111:176-81.

13. Ehrenstein V, Andersen SL, Qazi I, et al. Tenosynovial Giant Cell Tumor: Incidence, Prevalence, Patient Characteristics, and Recurrence. A Registry-based Cohort Study in Denmark. J Rheumatol 2017;44:1476-83. 
14. Houdek MT, Wyles CC, Rose PS, et al. High Rate of Local Recurrence and Complications Following Total Knee Arthroplasty in the Setting of Synovial Chondromatosis. J Arthroplasty 2017;32:2147-50.

15. Tibbo ME, Wyles CC, Rose PS, et al. Long-Term Outcome of Hip Arthroplasty in the Setting of Synovial Chondromatosis. J Arthroplasty 2018;33:2173-6.

16. Al-Ibraheemi A, Ahrens WA, Fritchie K, et al. Malignant Tenosynovial Giant Cell Tumor: The True "Synovial Sarcoma?" A Clinicopathologic, Immunohistochemical, and Molecular Cytogenetic Study of 10 Cases, Supporting Origin from Synoviocytes. Mod Pathol 2019;32:242-51.

17. Agaram NP, Zhang L, Dickson BC, et al. A molecular study of synovial chondromatosis. Genes Chromosomes Cancer 2020;59:144-51.

18. Nakayama R, Jagannathan JP, Ramaiya N, et al. Clinical characteristics and treatment outcomes in six cases of malignant tenosynovial giant cell tumor: initial experience of molecularly targeted therapy. BMC Cancer 2018;18:1296.

19. Alexiev BA, Tumer Y, Yang GY. Malignant tenosynovial giant cell tumor with CDKN2A/B genomic alteration: a histological, immunohistochemical, and molecular study. Hum Pathol 2017;63:144-8.

20. Biazzo A, Confalonieri N. Synovial chondrosarcoma. Ann Transl Med 2016;4:280.

21. Urwin JW, Cooper K, Sebro R. Malignant Transformation of Recurrent Synovial Chondromatosis: A Case Report and Review. Cureus 2019;11:e5839.

22. McCarthy C, Anderson WJ, Vlychou M, et al. Primary synovial chondromatosis: a reassessment of malignant potential in 155 cases. Skeletal Radiol 2016;45:755-62.

23. Ottaviani S, Ayral X, Dougados M, et al. Pigmented villonodular synovitis: a retrospective single-center study of 122 cases and review of the literature. Semin Arthritis Rheum 2011;40:539-46.

24. Lei P, Sun R, Liu H, et al. Prognosis of Advanced Tenosynovial Giant Cell Tumor of the Knee Diagnosed During Total Knee Arthroplasty. J Arthroplasty 2017;32:1850-5.

25. de Sa D, Horner NS, MacDonald A, et al. Arthroscopic surgery for synovial chondromatosis of the hip: a systematic review of rates and predisposing factors for recurrence. Arthroscopy 2014;30:1499-1504.e2.

26. Elsherif MA, Wenger DE, Vaubel RA, et al. NerveAdherent Giant Cell Tumors of Tendon Sheath: A New Presentation. World Neurosurg 2016;92:583.e19-24.

27. Algazwi DAR, Gartner LE, Manohara R, et al. Synovial
Chondromatosis of the Shoulder Causing Nerve

Compression. J Clin Rheumatol 2020;26:e289-90.

28. Kim SW, Choi JH. Synovial chondromatosis presenting with lumbar radiculopathy. Spine (Phila Pa 1976) 2009;34:E414-7.

29. Zhao G, Wang J, Xia J, et al. The predictive value of preoperative neutrophil-lymphocyte ratio (NLR) on the recurrence of the local pigmented villonodular synovitis of the knee joint. BMC Musculoskelet Disord 2018;19:339.

30. García-Ortega DY, Álvarez-Cano A, Sánchez-Llamas LA, et al. Neutrophil/lymphocyte ratio is associated with survival in synovial sarcoma. Surg Oncol 2018;27:551-5.

31. Cheng Y, Mo F, Pu L, et al. Pretreatment Inflammatory Indexes as Prognostic Predictors of Survival in Patients Suffering From Synovial Sarcoma. Front Oncol 2019;9:955.

32. Zhang J, Zhang HY, Li J, et al. The elevated NLR, PLR and PLT may predict the prognosis of patients with colorectal cancer: a systematic review and meta-analysis. Oncotarget 2017;8:68837-46.

33. Kaida T, Nitta H, Kitano Y, et al. Preoperative platelet-tolymphocyte ratio can predict recurrence beyond the Milan criteria after hepatectomy for patients with hepatocellular carcinoma. Hepatol Res 2017;47:991-9.

34. Vougiouklakis T, Shen G, Feng X, et al. Molecular Profiling of Atypical Tenosynovial Giant Cell Tumors Reveals Novel Non-CSF1 Fusions. Cancers (Basel) 2019;12:100.

35. Shi J, Zheng J, Zhou X, et al. Risk Factors Associated With Postoperative Recurrence in Patients With Tenosynovial Giant Cell Tumor of the Hand: A Retrospective Cohort Study. Ann Plast Surg 2019;83:523-8.

36. Tsukamoto S, Zucchini R, Staals EL, et al. Incomplete resection increases the risk of local recurrence and negatively affects functional outcome in patients with tenosynovial giant cell tumor of the hindfoot. Foot Ankle Surg 2020;26:822-7.

37. Ozben H, Coskun T. Giant cell tumor of tendon sheath in the hand: analysis of risk factors for recurrence in 50 cases. BMC Musculoskelet Disord 2019;20:457.

38. Mastboom MJL, Staals EL, Verspoor FGM, et al. Surgical Treatment of Localized-Type Tenosynovial Giant Cell Tumors of Large Joints: A Study Based on a MulticenterPooled Database of 31 International Sarcoma Centers. J Bone Joint Surg Am 2019;101:1309-18.

39. Mastboom MJL, Palmerini E, Verspoor FGM, et al. Surgical outcomes of patients with diffuse-type tenosynovial giant-cell tumours: an international, 
retrospective, cohort study. Lancet Oncol 2019;20:877-86.

40. Scheer M, Dantonello T, Hallmen E, et al. Synovial Sarcoma Recurrence in Children and Young Adults. Ann Surg Oncol 2016;23:618-26.

41. Ferrari A, Chi YY, De Salvo GL, et al. Surgery alone is sufficient therapy for children and adolescents with lowrisk synovial sarcoma: A joint analysis from the European paediatric soft tissue sarcoma Study Group and the Children's Oncology Group. Eur J Cancer 2017;78:1-6.

42. El Beaino M, Araujo DM, Gopalakrishnan V, et al. Prognosis of T1 synovial sarcoma depends upon surgery by oncologic surgeons. J Surg Oncol 2016;114:490-4.

43. Garvin S, Oda H, Arnesson LG, et al. Tumor cell expression of CD163 is associated to postoperative radiotherapy and poor prognosis in patients with breast cancer treated with breast-conserving surgery. J Cancer Res Clin Oncol 2018;144:1253-63.

44. Lin CY, Lovén J, Rahl PB, et al. Transcriptional amplification in tumor cells with elevated c-Myc. Cell 2012;151:56-67.

45. Nagy N, Bronckart Y, Camby I, et al. Galectin-8 expression decreases in cancer compared with normal and dysplastic human colon tissue and acts significantly on human colon cancer cell migration as a suppressor. Gut 2002;50:392-401.

Cite this article as: Wang W, Long MM, Wei CJ, Cui XW, Ren JY, Gu YH, Li QF, Dai SD, Gu B, Wang ZC. Clinical comparison of tenosynovial giant cell tumors, synovial chondromatosis, and synovial sarcoma: analysis and report of 53 cases. Ann Transl Med 2021;9(13):1059. doi: 10.21037/atm-21542
46. Nguyen TT, Schwartz EJ, West RB, et al. Expression of CD163 (hemoglobin scavenger receptor) in normal tissues, lymphomas, carcinomas, and sarcomas is largely restricted to the monocyte/macrophage lineage. Am J Surg Pathol 2005;29:617-24.

47. Kondo R, Akiba J, Hiraoka K, et al. Malignant diffuse-type tenosynovial giant cell tumor of the buttock. Pathol Int 2012;62:559-64.

48. Berger I, Weckauf H, Helmchen B, et al. Rheumatoid arthritis and pigmented villonodular synovitis: comparative analysis of cell polyploidy, cell cycle phases and expression of macrophage and fibroblast markers in proliferating synovial cells. Histopathology 2005;46:490-7.

49. Demir D, Yaman B, Anacak Y, et al. Prognostic significance of bcl-2, c-myc, survivin and tumor grade in synovial sarcoma. Turk Patoloji Derg 2014;30:55-65.

50. Nissen MJ, Boucher A, Brulhart L, et al. Efficacy of intraarticular bevacizumab for relapsing diffuse-type giant cell tumour. Ann Rheum Dis 2014;73:947-8.

51. Cassier PA, Italiano A, Gomez-Roca CA, et al. CSF1R inhibition with emactuzumab in locally advanced diffusetype tenosynovial giant cell tumours of the soft tissue: a dose-escalation and dose-expansion phase 1 study. Lancet Oncol 2015;16:949-56. 